\title{
Performance Evaluation of Edge Detection Techniques for Fractal Images
}

\begin{abstract}
Edge detection is a method that is used to spot the outline of an object or the boundaries between two objects. For recovering or extracting information from an image edge detection is often the primary step. Edge detection plays a momentous part in image processing. This paper lays out a comparison of several edge detection methods applied to fractal images. Fractals are the never-ending patterns, which have non-regular geometric shapes. For a few fractal images, measures like Mean square error (MSE) and Peak Signal to noise ratio (PSNR) are been calculated to point out the comparison between various edge detection methods.
\end{abstract}

Index Terms - Edge Detection, Fractal Image, MSE, PSNR

\section{INTRODUCTION}

Edge detection is utilized for the extraction of data in addition to image segmentation in areas like image processing and computer vision. The edge of an image is a sudden change of discontinuities. Edges generally arise on the border between the objects or images. Generally, edges are classified as Horizontal edges, Vertical edges, and Diagonal edges [1]. Here the question arises why we'd wish to detect the edge of an image? The solution to the present question is, as the maximum form of information of an image is surrounded in edges. So first the edge of the image is detected using edge detection methods and then the area of the image which contains edges are been enhanced, this may increase the sharpness of the image, and the also the image will become clean. The main reason for edge detection is to supply a stroke of a scene and to extract significant features from the edge of the image just like the corner, lines curves, etc [1]. The edges of an image are modelled in step with their intensity. The images edges of an image are modelled as [2].

- Step edge: the intensity of an image suddenly changes from one value on one side of the cut-off to the opposite value on the other side

- Ramp edge: It is a step edge where the change in intensity of an image is not immediate but arises over a finite distance.

Manuscript revised on July 05, 2020 and published on July 10, 2019 Priyanka Kujur, SUIIT, Sambalpur University, Odisha, India
- Ridge edge: the intensity of an image suddenly changes value yet again returns to the initial value within some short distance.

- Roof edge: It is a ridge edge where the intensity changes of an image is sudden but arises over a finite distance.

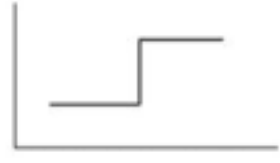

(a)

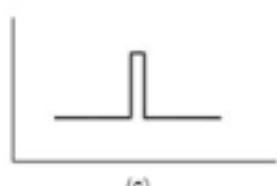

(c)

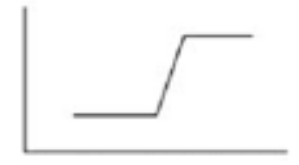

(b)

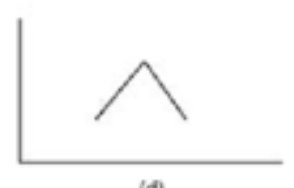

(d)
Figure 1:Figure shows (a)Step Edge (b) Ramp Edge (c) Ridge Edge (d) Roof Edge

Comparative study of several edge detection procedures is been found out and exposed in this paper. The edge detection methods include are Canny, Roberts, Sobel, Prewitt, and Laplacian edge detector. These methods are accustomed to detect edges of a fractal image. Fractals are the categories of shapes that we see in natures like the construction of trees, mountains, rocks, the formation of clouds, etc. Fractal could also be thought of as never-ending patterns. Fractal is derived from a Latin name fractus, which means an uneven surface like that of broken stone. So, fractal images are the non-regular symmetrical shape that has an identical degree of non-regularity.

This paper contains following more sections, section 2 describes the related works done by different authors. Section 3 describes the essential methods used for the comparative study of edge detection. Section 4 shows the result and analysis of the comparative study and section 5 winds up the paper with a conclusion.

\section{BACKGROUND STUDY}

Edge detection plays a significant role in image processing because it decreases the amount of data needed to be managed. It also gives important knowledge concerning the form of objects. Djemel Zious and Salvatore Tabbone on their paper proposed a summary of research in edge detection, edge definition, properties of detectors, the methodology of edge detection, the mutual influence between edges and detectors, and existing edge detectors and their implementation [3]. D. Poobathy and Dr.R. Manicka 
Chezian in their paper showed MSE and PSNR values of some edge detector method and also the time required by each method to detect the edge [4]. Tzu-Heng Henry Lee in his paper, Edge detection analysis shows quick learning of the elemental ideas of the edge detection operations, theories used for various detecting edges, and some simple Matlab functions which also show the simulation results for detecting edge [5]. T.Dharani, I.Laurence Aroquiaraj, and V.Mageshwari in their paper showed the comparison of detectors and algorithms that supports the Content-Based Image Retrieval process. This process is been applied to heterogeneous images [6]. Aborisade, D.O in their paper developed an edge detection algorithm that is based on fuzzy logic. This algorithm is used to find out edges for digital images at each pixel, it uses 3 filters i.e. low-pass, high-pass, and edge enhancement filter through a spatial convolution process [7]. M. Khairudin and Dessy Irmawati in their paper showed the comparison of three edge detection methods using USG [8]. Yi Zhang, Xiaoyuan Han, Han Zhang, Liming Zhao in their paper proposed an algorithm that combines improved Sobel, Canny, and LoG operator. This algorithm optimizes the results in order to resolve various measures which include the following low edge localization accuracy, poor noise, and edge detection continuity of Sobel operator [9]. You-Yi Zheng, Ji-lai Rao, and Lei Wu provide two methods: Canny operator and mathematical morphology in their paper, which synopses comparatively good image edge detection methods and delivers a reference for a few detection process which requires smaller edge width in practical application [10]. Dong Xiaoheng and Li Minghang, Miao Jiashu in their work proposed an edge detection operator. This operator works for detecting edges of an image which includes the underwater target image [11]. Langbein Zhang and Lifeng Xi proposed an edge detection method which is absolutely different from the other detection techniques in terms of self-similarity of fractal compression [12]. Klaus D.Toennies and Julia A.Schnabel showed edge detection methods that are based on the local fractal dimension and use Sobel and Laplace operators [13]. Muthukrishnan.R and M.Radha in their paper made a trial to check the performance of maximum used edge detection techniques for segmentation of image and also compared those techniques using MATLAB software [14]. Dharampal and Vikram Mutneja in their paper introduced standard edge detection techniques like Prewitt, Laplacian, Canny, Sobel, Roberts. Additionally, they discussed about Fuzzy logic [15].

\section{METHODS AND ITS COMPARATIVE STUDY}

There are various ways for edge detection, however, they are grouped into two categories Gradient and Gaussian. In a Gradient-based method, the first derived function of an image is found out to detect edge whereas, within the Gaussian based methods, the edges are detected by taking the second-order derivative expression which encompasses a zero crossing.
- Gradient Based Edge Detection: In the gradient-based method, the edge of an image is detected by seeking out the foremost maximum and minimum values of the primary derived function. The gradient-based approach is additionally called a mask in digital images [15]. In image processing, the first-order derivatives are achieved using the magnitude of the gradient.

- Gaussian Based Edge Detection: The Gaussian technique hunts for zero crossings within the second derived function to look out for edges. The edge of the image has a 1-D sort of a gradient, so the location of the image can be emphasized by calculating the derivatives for the image.

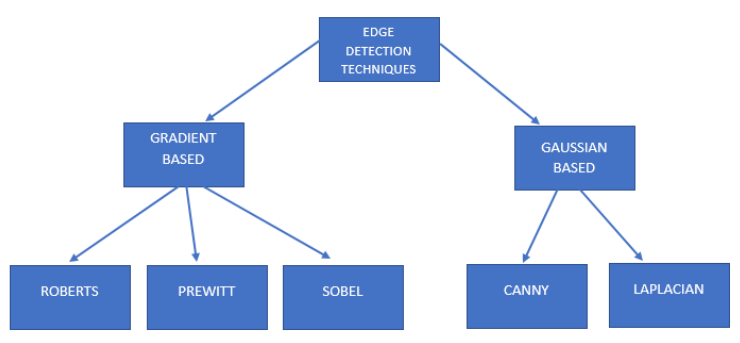

Figure 2:Techniques used for Edge detection

\section{Roberts Edge Detector:}

The Roberts edge detector was introduced by Lawrence Roberts in 1965. It computes a 2-D spatial gradient measurement on an image that is quick to compute. This process is employed to spotlight the regions having a high spatial frequency that frequently correspond to edges. The input given to the operator is a grayscale image. Pixel values in every point represent the complete magnitude of the spatial gradient of the input image at that point. This operator involves a pair of $2 \mathrm{X} 2$ convolution kernels as shown below. In this detector, a given kernel is nothing but a $90^{\circ}$ rotation of another kernel. These kernels are distinctly applied to the input images, to yield distinct measurements of the gradient component in each orientation (Gx and Gy) [16].

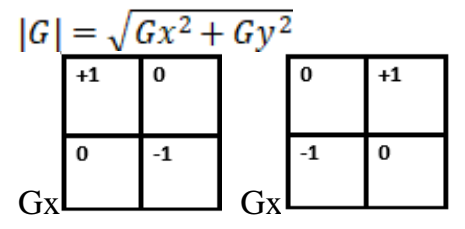

\section{Sobel Edge Detector:}

The Sobel edge detector was proposed by Sobel in 1970. This detector is extremely almost like a Prewitt edge detector. It's like surrounded derivatives that is used for edge detection. Sobel edge detection is used to calculates edge or detect an edge in both horizontal and vertical directions. The Sobel technique is used to perform a 2-dimensional symmetrical ramp quantity on an image and highlight regions having a 
high computative frequency that corresponds to edges. Generally, it is used to find the complete inclined magnitude at each point for $\mathrm{n}$ different grayscale image. This operator comprises a pair of $3 \mathrm{X} 3$ convolution kernels as shown below. In this detector, a given kernel is nothing but a $90^{\circ}$ rotation of another kernel. These kernels could be distinctly given to input images, which results as a distinct dimension of the gradient component in an individual perspective (Gx and Gy) The magnitude of these gradients is given as [16]:

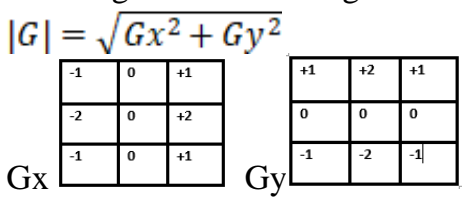

\section{Prewitt Edge Detector:}

The Prewitt detector was introduced by Rafael C. Gonzalez in the year 1970. Prewitt operator is employed for detecting edges horizontally and vertically. Prewitt is the exact method to guess the magnitude and alignment of an edge. This detector is calculable within the $3 \times 3$ nearest neighbours for eight different directions. All the eight different masks are been found out and one of the masks is been selected. This operator also turns the image with a low, distinguishable, and integer-valued filter in the horizontal and vertical direction and is therefore comparatively reasonable regarding computations [16].

$h 1=\left[\begin{array}{ccc}1 & 1 & 1 \\ 0 & 0 & 0 \\ -1 & -1 & -1\end{array}\right] h 2=\left[\begin{array}{lll}-1 & 0 & 1 \\ -1 & 0 & 1 \\ -1 & 0 & 1\end{array}\right]$

\section{Canny Edge Detector:}

In the Canny edge detection method, Gaussian functions is been used for smoothing the image where the maximum and the minimum value of the primarily derived function coincides with each other. This means the primary derived function both the maximum and minimum points, one has a dramatic change of gray-scale and other having a slight change of gray-scale corresponds to the second derived function also known as the zero-crossing point. These two measures are used to distinguish strong edges and weak edges.

\section{Laplacian Edge Detector:}

Laplacian is also a second-order derivative mask-like canny operator that is used to detect edges in an image. It is further categorized as positive and negative Laplacian. Generally, this operator takes a single gray level image as input and produces another gray level image as output. For smoother transitions, computing the second derived function as well as to perceive when this second derived function crosses zero is advantageous. If the second derived function crosses zero, it means that the location matches a maximum value and consequently this pixel location is considered as an edge. This method is additionally called as the zero-crossing edge detection technique.
So, a common mask operator used for the estimation of the second derived function is the Laplacian operator. The Laplacian $\mathrm{L}(\mathrm{x}, \mathrm{y})$ of an image with pixel intensity values $\mathrm{I}(\mathrm{x}, \mathrm{y})$ is given by $[16]$ :

$L(x, y)=\frac{\partial^{2} I}{\partial x^{2}}+\frac{\partial^{2} I}{\partial Y^{2}}$

Since the input image is signified as a group of discrete pixel values, first we need to seek out a discrete convolution kernel that is somewhat similar to the second derived function. Three commonly used small kernels are shown below

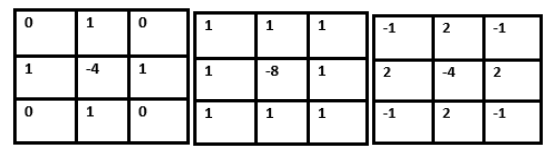

\section{RESULT AND ANALYSIS}

Four fractal images are been used for the comparative study of some edge detection techniques, like Mandelbrot, Circular Julia, Random, and Morphing. These fractal images are been given as input to various edge detection techniques using python (Version 3.8). The result of these operators is been compared by calculating various measures. These measures include Mean square error (MSE) and Peak Signal to noise ratio (PSNR) values.

Mean square error (MSE) is a parameter used to figure out the quality of the resultant image. If the value of MSE for the resultant image after application of the edge detection method is less, this means the quality of the resultant image is better.

$M S E=\frac{1}{R C} \sum_{X=1}^{R} \sum_{Y=1}^{C}\left[f(x, y)-f^{\prime}(x, y)\right]^{2}$

Where $\mathrm{f}(\mathrm{x}, \mathrm{y})$ is considered as the original input image, $\mathrm{f}^{\prime}(\mathrm{x}$, $\mathrm{y}$ ) is considered as the resultant image and $\mathrm{R}, \mathrm{C}$ are the dimensions of the images.

PSNR is a measure that finds the ratio between the sizes of the input image to the square of the value of Mean Square Error (MSE). If the value of PSNR is high, this means the quality of the resultant image is of better quality.

$P S N R=10 \log _{10}\left[\frac{R * C}{M S E}\right]$

Where, $\mathrm{RxC}$ is the size of the original or the input image.

Given below are the fractal images that are been used and the result extracted from different edge detection techniques. 
International Journal of Research in Advent Technology, Vol.8, No.6, June 2020

$$
\text { E-ISSN: 2321-9637 }
$$

Available online at www.ijrat.org

Table 1:MSE values calculated for different fractal images using various techniques.

\begin{tabular}{|l|l|l|l|l|l|}
\hline TECHNIQUES & CANNY & ROBERTS & PREWITT & SOBEL & LAPLACIAN \\
\hline MORPHING & 0.0093 & 0.0068 & 0.0055 & 0.0056 & 0.0062 \\
\hline MANDELBROT & 0.0208 & 0.0191 & 0.0196 & 0.0196 & 0.0205 \\
\hline $\begin{array}{l}\text { CIRCULAR } \\
\text { JULIA }\end{array}$ & 0.0201 & 0.0174 & 0.0144 & 0.0139 & 0.0158 \\
\hline RANDOM & 0.0201 & 0.0186 & 0.0177 & 0.0175 & 0.0174 \\
\hline
\end{tabular}

Table 2:PSNR values calculated for different fractal images using various techniques.

\begin{tabular}{|l|l|l|l|l|l|}
\hline TECHNIQUES & CANNY & ROBERTS & PREWITT & SOBEL & LAPLACIAN \\
\hline MORPHING & 44.36 & 45.69 & 46.42 & 46.52 & 46.14 \\
\hline MANDELBROT & 40.87 & 41.24 & 41.14 & 41.13 & 40.92 \\
\hline $\begin{array}{l}\text { CIRCULAR } \\
\text { JULIA }\end{array}$ & 41.02 & 41.65 & 42.46 & 42.60 & 42.06 \\
\hline RANDOM & 41.02 & 41.35 & 41.58 & 41.62 & 41.65 \\
\hline
\end{tabular}
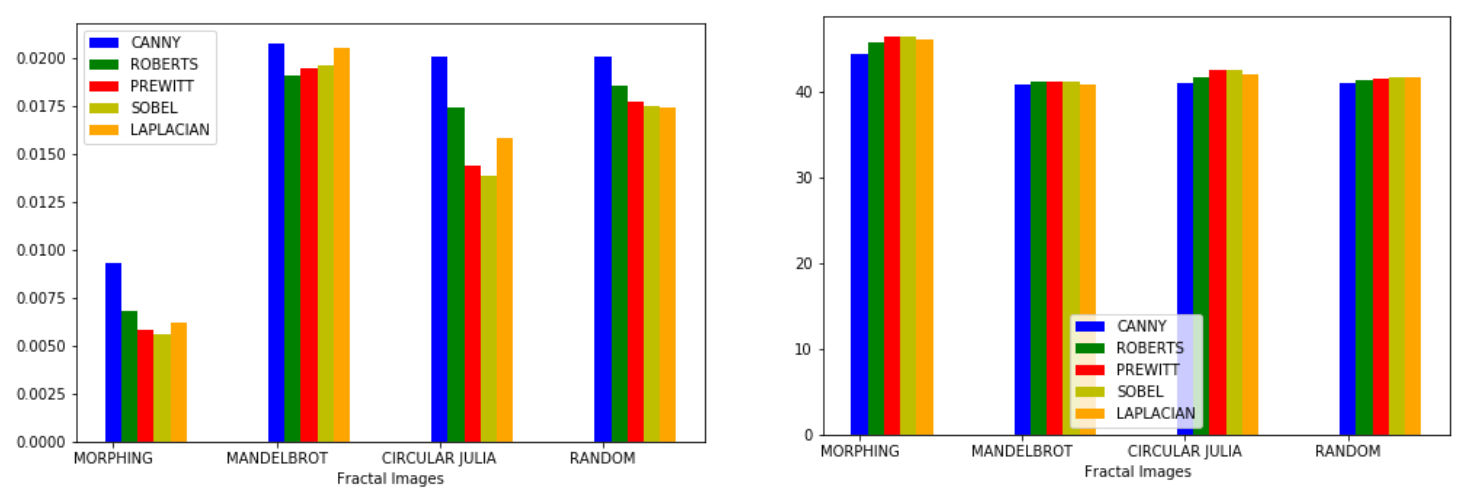

Figure 3:Graphical representation for (a)MSE and (b)PSNR value calculated for various fractal images for various edge detection techniques.

Table 3, shows the four original fractal images like Morphing, Mandelbrot, Circular Julia, and Random along with the result, after applying edge detection methods. Five edge detection methods used here are Canny, Roberts, Prewitt, Sobel, and Laplacian. Table1 displays the MSE value and Table 2 displays the PSNR value of the input Fractal images in different edge detection methods. Figure 3 shows the graphical representation of the calculated MSE and PSNR values. 
International Journal of Research in Advent Technology, Vol.8, No.6, June 2020

$$
\text { E-ISSN: 2321-9637 }
$$

Available online at www.ijrat.org

Table 3:Original fractal images with result after using various methods.

\begin{tabular}{|c|c|c|c|c|}
\hline $\begin{array}{c}\text { FRACTAL } \\
\text { IMAGES }\end{array}$ & MORPHING & MANDELBROT & CIRCULAR JULIA & RANDOM \\
\hline \multicolumn{5}{|l|}{ ORIGINAL } \\
\hline \multicolumn{5}{|l|}{ CANNY } \\
\hline \multicolumn{5}{|l|}{ ROBERTS } \\
\hline \multicolumn{5}{|l|}{ PREWITT } \\
\hline \multicolumn{5}{|l|}{ SOBEL } \\
\hline \multicolumn{5}{|l|}{ LAPLACIAN } \\
\hline
\end{tabular}

\section{Conclusion}

Image Processing and Computer Vision is the area where edge detection plays a vital role. So, to extract information and image segmentation we need to detect the edge of the image. To detect the edge of an image various edge detection techniques are been used. In this paper, the working of some edge detection techniques like Roberts, Prewitt, Sobel,
Canny, and Laplacian methods are laid out where Roberts, Prewitt, and Sobel are gradient-based methods whereas Canny and Laplacian are Gaussian based. The comparison of MSE and PSNR values of the resultant images are also been calculated in order to find out which edge detection method gives a better result. This paper will work like a review for the ones who are new to this field. 
International Journal of Research in Advent Technology, Vol.8, No.6, June 2020

E-ISSN: 2321-9637

Available online at www.ijrat.org

\section{REFERENCES}

[1] "https://www.tutorialspoint.com/dip/concept of edge detection.htm," tutorial point, [Online]. Available: https://www.tutorialspoint.com/dip/concept of edge detection.htm.

[2] J. Trucco, "Edge detection," in Trucco, Chapt 4 AND Jain et al., Chapt 5.

[3]D. Ziou, "Edge Detection Technique-An Overview," researchgate, 1998.

[4]D. Poobathy and R.Manika Chezian, "Edge Detection Operators: Peak Signal to Noise Ratio Based Comparision," 2014.

[5] Tzu-Heng Henry Lee, "Ege detection Analysis," 2007.

[6]T.Dharani, I.Laurence Aroquiaraj and V.Mageshwari, "Comparative Analysis of Edge Detection Algorithm Based on Content-Based Image Retrieval with Heterogeneous Images," International Journal of Computational Intelligence and Informatics, vol. 5, 2016.

[7] Aborisade, "Fuzzy Logic Based Digital Image Edge Detection," vol. 10, 2010.

[8] M.Khairudin and Dessy Irmawati, "Comparison Methods of Edge Detection for USG Images," in Proc. of 2016 3rd Int. Conf. on Information Tech., Computer, and Electrical Engineering (ICITACEE), Semarang, Indonesia, 2016.

[9] X. H. H. Z. L. Z. Yi Zhang, " Edge Detection Algorithm of Image Fusion Based on Improved Sobel Operator," IEEE, 2017.

[10] J.-1. R. L. W. You-yi Zheng, " Edge Detection Methods in Digital Image Processing," in The 5th International Conference on Computer Science $1 \&$ Education Hefei, China, 2010.

[11] L. M. M. J. W. Z. Dong Xiaoheng, " Edge Detection Operator for Underwater Target Image," in 3rd IEEE International Conference on Image, Vision and Computing, 2018.

[12] Liangbin Zhang and Lifeng Xi, " A Novel Image Edge Detection Using Fractal Compression," Springer, 2007.

[13] J. A. Klaus D.Toennies, " Edge Detection Using the Local Fractal Dimension," IEEE, 1994.

[14] M. a. M.Radha, " EDGE DETECTION TECHNIQUES FOR IMAGE SEGMENTATION," International Journal of Computer Science I\& Information Technology (IJCSIT), vol. 3, 2011.

[15] D. a. V. Mutneja, "Methods of Image Edge Detection: A Review," J Electr Electron Syst, 2015.

[16] G. S. A. K. Pooja Sharma, "Different Techniques Of Edge Detection In Digital Image Processing," International journal of Engineering Research and Applications (IJERA), vol. 3, no. 2248-9622, 2013.

\section{AUTHORS PROFILE}

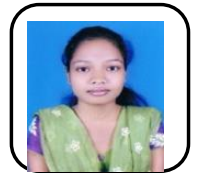

Priyanka Kujur received the bachelor's degree in Information Science and Telecommunication and master's degree in Computer Science from Ravenshaw University,

Cuttack, Odisha, India. She received the professional postgraduate engineering master degree in Computer Science and Engineering from SUIIT, Sambalpur University, Burla, Odisha, India. 